\title{
Determination of Arsenic and Health Risk Assessment in the Ground Water of Sindh, Pakistan
}

\author{
ZULFIQAR ALI BHATTI*, KHADIJA QURESHI*, INAMULLAH BHATTI*, IMRAN NAZIR UNAR*, \\ AND MOHAMMAD YAR KHUHAWAR** \\ RECEIVED ON 14.02.2017 ACCEPTED ON 29.05.2017
}

\begin{abstract}
As (Arsenic) is one of the lethal element present at the various locations of the world, putting human beings in danger by polluting the water. Arsenic Kit and atomic absorption spectrometer were used to determine As in ground water of Sindh province, Pakistan. Twenty-Four (24) districts both on the left and right bank of RI (River Indus) were analyzed. It was observed from the results that highest As concentration $200 \mathrm{ppb}$ (parts per billion) i.e. above the WHO (World Health Organization) limit (10 ppb) was observed in Sakrand, district Shaheed Benazirabad followed by Hala, Matairi, TMK (Tando Mohammad Khan) and Nasarpur regions. It was further found that ground water of regions on the left bank of RIwas more contaminated than the right bank. Contour map was created using OriginPro and coordinate systems to highlight the elevated arsenic in the studied area. HRA (Health Risk Assessment) of these areas was carried out to calculate EDI (Estimated Daily Intake), TQH (Target Hazard Quotient) and CR (Cancer Risk). $45 \%$ of the total ground water samples analyzed were above the permissible limit for As in water and mostly these are located on the left bank of RI.
\end{abstract}

The local wells in Sindh have never been tested for metal concentration former to use. These results provide baselines for researchers, NGO's (Non-Governmental Organizations) and government to apply arsenic treatment technologies in those areas.

Key Words: Arsenic, Groundwater, River Indus, Sindh, Atomic Absorption Spectrometer, Health Risk Assessment.

\section{INTRODUCTION}

A $\mathrm{S}$ is a hazardous element which is present in ground water in the form of As(III) and As(V). Its presence in the earth's crust is $0.00021 \%$. Illness characteristic of long-term arsenic exposure comes in the form of skin cancer, bladder cancer, inactivation of an enzyme system in the body, black foot disease, and obesity [1-3]. As contact can also cause other health consequences such as gastrointestinal irritation, diabetes, less number of white and red blood cells, hypertension, blood vessels rupture and abnormal heart beats [4]. Baig

Corresponding Author (E-Mail: zulfiqar.bhatti@faculty.muet.edu.pk)

* Department of Chemical Engineering, Mehran University of Engineering \& Technology, Jamshoro.

** Institute of Advanced Research Studies in Chemical Sciences, University of Sindh, Jamshoro.

Mehran University Research Journal of Engineering \& Technology, Volume 36, No. 4, October, 2017 [p-ISSN: 0254-7821, e-ISSN: 2413-7219] 
et. al. [3] performed a study of Gambat town, Sindh and found people suffering from chest infections, pain in the whole body, cramps in legs due to As contamination in water.

Naturally occurring arsenic is known to trickle from contaminated soils. Thus, contact of the general population with arsenic is mainly through the ingestion of groundwater, which comes across As-bearing soils and minerals. Pakistan is suffering from the toxic effects of arsenic as other countries such as Bangladesh, India, Thailand, Taiwan, China, Japan, USA, Canada, Hungary and Ghana [5].

The high level of As species in ground water is due to the dissolution of As compounds coming from the Himalayas through RI and settled down throughout the years and then introduced into ground water by geothermal, hydrological and biochemical factors [6]. In Pakistan, a number of people consume ground water for their drinking, domestic purposes as clean river water is not accessible to them. As toxicity is mostly found in ground water and transported to human beings by ingestion, vegetables, crops, fruits and fishes. The authors in a research showed that the Mancharlake near town Sehwan, Sindh was contaminated with arsenic and the daily intake of As by people is 241-390 $\mu \mathrm{g}$ As/4L [5].

The present study was designed to investigate As in drinking water of Sindh province on both left and right banks of RI.

\section{MATERIALS AND METHOD}

\section{$2.1 \quad$ Materials}

Ground water samples were collected from various areas of Sindh Province, Pakistan and analyzed in a water quality laboratory, Department of Chemical Engineering, Mehran University of Engineering \& Technology, Jamshoro,
Pakistan. All sample bottles (well-stoppered polyethylene plastic) were rinsed with clean water, then filled completely with sample water and tagged properly with a location. Analysis of water samples was performed by using the Arsenic test Kit (Merck, Germany) and AAS (Atomic Absorption Spectrometer). The arsenic kit is a mobile analytical tool that is used in the field to check the arsenic in water. AAS was used to analyze 106 ground water samples, whereas kit method was applied to test 214 ground water samples.

Analytical grade chemicals were used throughout the research work. Distilled water was used during the experiments to prepare all the solutions. Distilled water was prepared in a water quality laboratory by Distilled water machine (Model No. BMS-4CE, Spain). All the glass wares were kept overnight in $5 \mathrm{M} \mathrm{HNO}_{3}$ and then rinsed with deionized water before use. Stock solutions of concentration $1000 \mathrm{ppb}$ As(III) were prepared by mixing arsenic standard solution of $100 \mathrm{mg} / \mathrm{L}$ As(III) (Inorganic Venture Company, USA) with distilled water. The stock solutions were used to prepare fresh experimental solutions as per need in clean plastic bottles and were kept away from light to avoid any change in chemical properties [7-9]. For the confirmation of reproducibility, the tests were replicated and the mean values were taken.

\subsection{Methods}

\subsubsection{Arsenic Kit method}

Water samples were analyzed by Arsenic kit (Merck, Germany) by using a calorimetric method with test strips. The arsenic kit reaction bottle was filled to the mark with $60 \mathrm{ml}$ sample water and the analysis was carried out as recommended by the kit manufacturer.

\subsubsection{Atomic Absorption Spectrometer}

Arsenic was analyzed in water samples by AAS (A Analyst 700, Perkin Elmer, USA), at a wavelength of

Mehran University Research Journal of Engineering \& Technology, Volume 36, No. 4, October, 2017 [p-ISSN: 0254-7821, e-ISSN: 2413-7219] 
193.7 nm, by the MHS-15 (Mercury Hydride System) technique. The energy source was the single-element hollow cathode lamp of arsenic working at $7.5 \mathrm{~mA}$. The AAS was attached to a computer and WinLab32 software used to receive and process analytical data. The solution of $1 \%$ potassium hydroxide $(\mathrm{KOH})$ was prepared. Then $3 \%$ sodium borohydride $\left(\mathrm{NaBH}_{4}\right)$ was prepared in $1 \% \mathrm{KOH}$ solution on Stirrer (Wisd, MSH-20, Korea). The chemicals were weighed using weighing balance (Adventurer, AR 3130, OHAUS, USA). 1.5\% HCl (Hydrochloric Acid) was prepared by appropriate dilution of $\mathrm{HCl}$ (37\%). The arsenic standards were prepared to calibrate the AAS. Then the ASS was used to analyze water samples and the results were obtained by Winlab 32 .

\subsubsection{Technique to Assess Human Health Risk}

The HRA of the left bank of RI areas is studied by calculating EDI, THQ and CR. EDI is calculated by using the following equation;

$$
\mathrm{EDI}=\mathrm{Cm} \times \frac{\mathrm{Dw}}{\mathrm{Bw}}
$$

Where $\mathrm{Cm}$ denotes metal concentration $(\mu \mathrm{g} / \mathrm{L})$ in water, Dw is the daily average intake of water (Lit/day, taken as $2 \mathrm{Lit} / \mathrm{day}$ ), and Bw is the body average weight ( $\mathrm{kg}$, taken as $72 \mathrm{~kg}$ ) [10].

The THQ was obtained by applying the following relationship;

$$
\mathrm{THQ}=\frac{\mathrm{EDI}}{\mathrm{RfD}}
$$

Where the oral toxicity reference doseRfD ( $\mu \mathrm{g} / \mathrm{kg}$-day) value for arsenic is (3.00E-01) [11].

Following relationship was used to calculate CR;
$\mathrm{CR}=\mathrm{EDI} \mathrm{x} \mathrm{CSF}$

In Equation (3) CSF ( $\mu \mathrm{g} / \mathrm{kg}$-day) is Cancer Slope Factor and its value for As is $1.5 \mathrm{E}+03$ ( $\mu \mathrm{g} / \mathrm{kg}$-day) [12-13].

\section{RESULTS AND DISCUSSION}

\subsection{Arsenic kit Method}

The As kit is a portable and easy tool method used to analyze As of 24 districts of Sindh Province. Out of 214 ground water samples, 182 were from localities on the left bank and 32 samples from the right bank of RI. All the sample areas were assigned codes as shown in Table 1.

The results of localities on the left bank are reported in Table 1. About $40 \%$ of the samples were exceeding the WHO safe limit for As in water, i.e. 10 ppb. The higher As levels were observed in ground water samples of Hala i.e. 10-180 ppb. The other areas with higher As concentrations were Sakrand, Matairi, Nasarpur, Pano Aqil and TMK.

The reasons for higher concentrations in the left bank of RI are the geological presence due to the deposition over the years from the Himalayas, thick population, intense farming, and the areas from where RI passed during the Holocene period such as Tando Allahyar and TMK from where the As ranges from 10-500 ppb [6].

Fig. 1 demonstrates the sample from the right bank of RI. The Dadu district samples showed higher As levels ranging between 0-60 ppb which is inconsistent with the earlier report of As in scalp and hair of children of Dadu district [14]. Apart from the natural deposition of arsenic in ground water other factors for As pollution are coal burning at brick industries, power plants in Jamshoro district [15]. Samples of Jamshoro and Kashmore ranged within permissible limit of $10 \mathrm{ppb}$.

Mehran University Research Journal of Engineering \& Technology, Volume 36, No. 4, October, 2017 [p-ISSN: 0254-7821, e-ISSN: 2413-7219] 
Determination of Arsenic and Health Risk Assessment in the Ground Water of Sindh, Pakistan

TABLE 1. GROUND WATER SAMPLE CODES ASSIGNED TO LOCATIONS OF SINDH, PAKISTAN AND ARSENIC KIT RESULTS

\begin{tabular}{|c|c|c|c|c|c|}
\hline Code & Location & $\mathrm{As}(\mathrm{ppb})$ & Code & Location & $\overline{\mathrm{As}(\mathrm{ppb})}$ \\
\hline BD1 & Hand Pump, NabiBuxBhurgri, Badin & 0 & ST3 & Abbasi Village, Sekhat & 40 \\
\hline BD2 & MuhammadNizamiMalhan, Matli, Badin & 0 & TM1 & Village SaeedPur, Burri Shah Karim, TMK & 3 \\
\hline BD3 & NabiBuxBhurgri, Badin & 0 & TM2 & Water Pond, Sajawal Road, TMK & 0 \\
\hline BD4 & NizamaniOtaq, Matli, Badin & 0 & TA1 & Mosque, Village Talpur, TandoAllahyar & 25 \\
\hline BD4 & Village Malhan, Matli, Badin & 0 & $\mathrm{TA} 2$ & Village Talpur, TandoAllahyar & 0 \\
\hline DK1 & Ghulab Shah Colony, Dharki & 0 & SR1 & Old Sukkur & 0 \\
\hline DK2 & Imam Bargah, Dharki & 5 & TM10 & SafeenaShifa, TMK & 30 \\
\hline DP1 & DaburiWell Water, Diplo & 0 & TM11 & SaqibTalpur, TMK & 5 \\
\hline DP2 & DileepSaqiHome, Diplo & 0 & TM12 & Tube Well Water (City), TMK & 50 \\
\hline DP3 & RafiqLangho, Diplo & 0 & TM13 & Village Gaja Mori, TMK & 0 \\
\hline DP4 & SaqiParo, Diplo & 0 & TM14 & Village GhulamHussainTalpur, TMK & 50 \\
\hline GK2 & Hand Pump, Yaro Lund, MirpurMathelo, Ghotki & 5 & TM3 & Bus Stop Near Ayobia Hotel, TMK & 60 \\
\hline GK3 & Motor Water, Yaro Lund, MirpurMathelo, Ghotki & 0 & TM4 & Gaja Mori, Sajawal Road, TMK & 0 \\
\hline HD1 & House No. 137, Latifabad, Hyderabad & 0 & TM5 & Govt: Girls High School, TMK & 40 \\
\hline HD2 & House No. 97, Latifabad, Hyderabad & 0 & TM6 & Govt: Girls School, TMK & 5 \\
\hline HD3 & Hussainabad, Police Colony, Hyderabad & 0 & TM7 & MadarsahJamiatmadinah, TMK & 20 \\
\hline HD6 & Latifabad, Hyderabad & 0 & TM8 & Majid Ali, Mir Mohalla, TMK & 6 \\
\hline HD7 & Miyani Road, Hyderabad & 0 & TM9 & SaeedPur, TalukaBulri Shah Karim, TMK & 100 \\
\hline HD8 & MohsinMemon, Citizen's Colony, Hyderabad & 5 & TB1 & 14 KM from GuniChowk, TandoBago & 10 \\
\hline HD9 & New Wahdat Colony, Hyderabad & 10 & TJ2 & RazaSaeed, Tando Jam & 15 \\
\hline IK1 & Govt: RO Plant, IslamKot & 0 & TT2 & Warial Khan Khoso, Jhirk, Thatta & 0 \\
\hline IK2 & Private RO Plant, IslamKot & 0 & UK3 & AkriPithoro, Umerkot & 10 \\
\hline IK3 & Village BhabinoBhell, UC SonalBhoh, Thar & 0 & HL1 & Abdul Aziz, KadhMohalla, Hala & 30 \\
\hline IK4 & Coal Gasification Site, IslamKot, Thar & 0 & HL10 & DargahMakhdomNoh,SalineWater, Pump 2, Hala & 40 \\
\hline KD1 & Mohammad Din, Kandiaro & 0 & HL11 & DargahMakhdom Noh,Sweet Water, Pump 1, Hala & 60 \\
\hline JD7 & Liaquat, Ward-2, Jhuddo & 0 & HL12 & DawoodMemonHome, KanarMohalla, Hala & 50 \\
\hline KP10 & Naib Ali, Tarkowado, Khairpur Mir's & 0 & HL13 & Dr. AR Memon Home, Hala & 35 \\
\hline KP13 & TarkoWado, Khaipur Mir’s & 0 & HL14 & Envious Girls Public School, KadhMohalla, Hala & 50 \\
\hline KP14 & Tube Well, TalukaNaro, Khairpur & 0 & HL15 & Faiz Mohammad, Hala New & 45 \\
\hline KP16 & Village Allah Dino Khuhro,Gambat,Khairpur & 45 & HL16 & Fareed Ahmed Home, PirFazalColony, Hala & 180 \\
\hline KP18 & Wandh, Khairpur Mir's & 10 & HL17 & Fazal, Hala New & 7 \\
\hline KGM1 & Jamesabad, KotGhulam Mohammad & 0 & HL18 & Govt: Girls High School, KadhMohalla, Hala & 5 \\
\hline KP3 & HadiBuxLeghari, ThariMirwah, Khairpur & 5 & HL19 & Haji Anwar, Kaka Mohalla, Hala & 40 \\
\hline KP4 & Hand pump, TalukaNaro, Khairpur & 5 & HL2 & Aftab Ansari, Hala & 25 \\
\hline KP6 & Jadowahan, Gambat, Khairpur & 25 & HL20 & Haji Faiz Mohammad Home, SiraiMohalla, Hala & 50 \\
\hline KP8 & Latif colony, Kairpur Mir’s & 5 & HL21 & Haji QamarudinHome, Kanar Mohall, Hala & 55 \\
\hline MT16 & HazoorBux Shop, Khyber Stop, Matairi & 30 & HL22 & Imam Bargah, Hala & 40 \\
\hline MH1 & Mithi & 0 & HL23 & Imdad Rajput, MohajirChowk, Hala & 25 \\
\hline MH2 & RO Water, Mithi & 0 & HL24 & JealArbabHome, ArbabMohalla, Hala & 70 \\
\hline MT10 & Macon-Washy, Matairi & 0 & HL25 & Khair Mohammad, PiniladhoColony, Hala & 30 \\
\hline MT11 & MatairiCity & 80 & HL26 & Khokhar Fridge Shop, BrohiMohalla, Hala & 10 \\
\hline MT12 & Matairi City, DargahPardehiPir & 100 & HL27 & MuhammadUsmanHome, PiniLadho Colony, Hala & 50 \\
\hline MT13 & Matairi Sugar Mills Officers Mess, Matairi & 110 & HL28 & MuhammadUsmanHome, PirFazal Colony, Hala & 100 \\
\hline MT14 & Matairi Sugar Mills RO Plant, Matairi & 120 & HL29 & MuhammadYousuf, TalibMola Road, Hala & 40 \\
\hline MT15 & Matairi Sugar Mills Supervisor mess, Matairi & 25 & HL3 & Ahmed KhalkaniHome, Kaka Mohalla, Hala & 50 \\
\hline MT16 & MianpotaMohalla, Matairi & 0 & HL30 & Mohammad Hussain, Kaka Mohalla, Hala & 25 \\
\hline MT17 & Mir Hassan Mohalla, Matairi & 5 & HL31 & Mohammad Usman, MohajirChowk, Hala & 20 \\
\hline MT18 & Sekhat, Matairi & 50 & HL32 & Mohammad, Hala New & 25 \\
\hline MT19 & ShameerJiWari, Matairi & 0 & HL33 & Mosque, Hala New & 50 \\
\hline MT2 & AbasPir, Highway Road, Matairi & 0 & HL34 & Naseer, Kaka Mohalla, Hala & 30 \\
\hline MT20 & Village BachalDetho, Near Matairi Sugar Mills & 100 & HL35 & Near Girls College, Hala & 25 \\
\hline MT21 & Village Bhambra, Matairi & 0 & HL36 & Near Imam Bargah, Hala & 40 \\
\hline MT22 & Village Shah Alam Shah Wasi, Matairi & 40 & HL37 & Old Hala & 10 \\
\hline MT23 & Village Vighio, Matairi & 0 & HL38 & Prof. Nafees Ahmed, KashigarMohalla, Hala & 40 \\
\hline
\end{tabular}

Mehran University Research Journal of Engineering \& Technology, Volume 36, No. 4, October, 2017 [p-ISSN: 0254-7821, e-ISSN: 2413-7219] 
Determination of Arsenic and Health Risk Assessment in the Ground Water of Sindh, Pakistan

\begin{tabular}{|c|c|c|c|c|c|}
\hline Code & Location & As(ppb) & Code & Location & $\mathrm{As}(\mathrm{ppb})$ \\
\hline MT3 & BurkatPota, Matairi & 0 & HL39 & Raja Fahad Home, Hala & 90 \\
\hline MT4 & Canteen, Matairi Sugar Mills & 0 & HL4 & Al Shifa Medical center,KadhMohalla, Hala & 50 \\
\hline MT5 & Graveyard, Matairi & 30 & HL40 & Raja FahadQureshi Agricultural Farm,Hala & 90 \\
\hline MT9 & Khyber, Matairi & 0 & HL41 & Rehan home,KashigarMohalla, Hala & 70 \\
\hline NF3 & Darpa, Tharushah, NausheroFeroz & 0 & HL42 & SaeedMemon, Hala New & 50 \\
\hline NF4 & KhudaBuxKalhoro, NausheroFeroz & 10 & HL43 & SarfarazQureshi, Mujawar colony, Hala & 10 \\
\hline NF6 & Tharushah, NausheroFeroz & 0 & HL44 & Shamsudin Book Store, Kanar, Hala & 10 \\
\hline NF7 & Village Allah Bachayo, NausheroFeroz & 3 & HL45 & Soomar Shah, Hala & 90 \\
\hline UK9 & Pump Water, Umerkot & 5 & HL46 & Sweet Shop, Hala & 13 \\
\hline NK1 & Naukot & 0 & HL47 & TahirYasirHome, TalibMola Road, Hala & 150 \\
\hline NP10 & Ward-6, Nasarpur & 15 & HL48 & TalibMola Colony, Hala & 100 \\
\hline NP2 & ArainMohallah, Nasarpur & 13 & HL49 & Tariq Road, Hala & 40 \\
\hline NP3 & Bus Stop, Nasarpur & 80 & HL5 & Alamgir, Kaka Mohalla, Hala & 50 \\
\hline NP7 & SikanderAlmaniHome, Nasarpur & 40 & HL50 & Tariq, Hala & 20 \\
\hline NP9 & Ward-4, Nasarpur & 35 & HL51 & Tariq, Hala New & 50 \\
\hline NF1 & ImdadHome Halani, NausheroFeroz & 60 & HL52 & Zameer, KanarMohalla, Hala & 45 \\
\hline NF2 & SajadHome Halani,NausheroFeroz & 25 & HL6 & Al-Hafeez Pathology, Hala & 40 \\
\hline NS3 & Aslam, ShaheedBenazirabad & 0 & HL7 & Allah Bux, ShahnawazMohalla, Hala & 10 \\
\hline NS4 & Habib College, ShaheedBenazirabad & 50 & HL8 & Arbani Street, Hala & 50 \\
\hline NS1 & Habib Sugar Mills ShaheedBenazirabad & 0 & HL9 & Cloth Factory, PiniladhoColony, Hala & 40 \\
\hline PA10 & Village Bhelar, PanoAqil & 35 & DD1 & AB Jamali, Johi, Dadu & 0 \\
\hline NS2 & Railway station ShaheedBenazirabad & 0 & DD4 & JohiCity, Pump 2, Dadu & 0 \\
\hline PA5 & Basheerabad,PanoAqil & 90 & DD11 & Water Well, JohiCity, Dadu & 0 \\
\hline PA6 & Dhandi, PanoAqil & 0 & DD8 & Village Allah Dad, Johi, Dadu & 5 \\
\hline PA8 & ManderGali, PanoAqil & 10 & DD2 & JohiCity Pump 1, Dadu & 10 \\
\hline PA9 & Shahi Bazar, PanoAqil & 2 & DD7 & Sample Pump 3, JohiCity, Dadu & 10 \\
\hline PA1 & Village Sanghi, PanoAqil & 5 & DD3 & JohiCity, Dadu & 60 \\
\hline PA2 & Village Sultan Pur, PanoAqil & 30 & JC1 & AKColony Jacobabad & 0 \\
\hline RH2 & Village Abello, Rohri & 0 & JC2 & PaichohaVillage, Jacobabad & 0 \\
\hline ST1 & Village Abbasi, Sekhat & 80 & JC3 & Village Pandhi, Jacobabad & 0 \\
\hline SK1 & Seed Plant, MajeedKeerio, Sakrand & 35 & JS2 & SajadAbbasi, Phatak, Jamshoro & 0 \\
\hline SK2 & Wasomal Stop, MajeedKeerio, Sakrand & 0 & JS3 & Syed YaseenHome, SUECH Society, Jamshoro & 0 \\
\hline ST2 & Village Abbasi, Sekhat & 50 & JS4 & Village Nawab Khan Khoso, Jamshoro & 0 \\
\hline SP1 & Society, Shahdadpur & 4 & JS6 & ZABhatti Home, SUECHS, Jamshoro & 0 \\
\hline SK10 & Jan Mohammad House,, Sakrand & 0 & JS1 & OwaisBhatti, Phatak, Jamshoro & 10 \\
\hline SK11 & KhadimHussainLakho, MajeedKeerio, Sakrand & 5 & KH1 & Haris, Upper Gizri, Clifton, Karachi & 0 \\
\hline SK12 & Mosque, MajeedKeerio, Sakrand & 0 & KM1 & Hindu Colony, Kashmore & 0 \\
\hline SK13 & MunawarJamali House, MajeedKeerio, Sakrand & 10 & KM3 & Town, Kashmore & 0 \\
\hline SK14 & Murtaza Agricultural Farm 1, Sakrand & 5 & KM4 & Village MohdHashim, Kashmore & 0 \\
\hline SK15 & Murtaza Agricultural Farm 2, Sakrand & 5 & KM2 & Guddu, Kashmore & 10 \\
\hline SK16 & Qasim Ansari House, MajeedKeerio, Sakrand & 0 & KT1 & Market Kotri & 0 \\
\hline SK17 & RazzaqKhokhar, MajeedKeerio, Sakrand & 8 & KT2 & Pathan Colony, Kotri & 0 \\
\hline SK18 & School Road, MajeedKeerio, Sakrand & 0 & LR4 & Hand Pump, Larkana & 0 \\
\hline SL1 & RasoolBuxTheboHome, Sajawal & 0 & LR9 & Ratodero, Larkana & 2 \\
\hline SK3 & Animal Hospital, MajeedKeerio, Sakrand & 5 & LR1 & Ali SherKhokhar, Larkana & 5 \\
\hline SK4 & Anwar, Sakrand & 0 & LR3 & Dhamrah, Larkana & 5 \\
\hline SK5 & AwaisMugheri home, MajeedKeerio, Sakrand & 25 & SH3 & Akbar Brohi, Shahdadkot & 0 \\
\hline SK6 & DargahWater, MajeedKerio, Sakrand & 10 & SH4 & Masan Mohalla, Shahdadkot & 0 \\
\hline SK7 & Drip No. 1 Water, MajeedKerio, Sakrand & 3 & SH5 & Saifullah Colony, Shahdadkot & 0 \\
\hline SK8 & Godown,MajeedKeerio, Sakrand & 50 & SH1 & Shahdadkot & 0 \\
\hline SK9 & Gulshan-e-Imdad, MajeedKeerio, Sakrand & 0 & SH2 & Tanwary Branch, Shahdadkot & 0 \\
\hline SR3 & Jamia Mosque, Bander Road, Sukkur & 80 & SR2 & NizamdinBhatti, GarhiYaseen, Shikarpur & 5 \\
\hline SG2 & Public Handpump, Sarhari, Sanghar & 0 & & & \\
\hline
\end{tabular}

Mehran University Research Journal of Engineering \& Technology, Volume 36, No. 4, October, 2017 [p-ISSN: 0254-7821, e-ISSN: 2413-7219] 


\subsection{Atomic Absorption Spectrometry}

AAS was used to analyze the samples from the left bank of RI and the results are shown in Fig. 2. The Fig. 2 is showing minimum and maximum As concentrations of the studied areas. Eighty-three (83) ground water samples were obtained from various locations of left bank RI. The highest As concentration observed was $204 \mathrm{ppb}$ at Godown, Majeed Keerio in Sakrand, district Shaheed Benazirabad. It was observed that $85 \%$ of the studied areas had arsenic above WHO limit, i.e. 10 ppb, whereas, $43 \%$ of the analyzed areas reported the level of arsenic above the Pakistan-EPA standard i.e. 50 ppb.

\subsection{Comparison of As Kit and AAS}

The As kit and AAS results are quite similar as shown by percent standard deviation (\% SD ( \pm ) ) in Table 2. The As kit and AAS (\% SD) results are comparable with a research work conducted on As detection of ground water at district Rahim Yar Khan, Punjab, Pakistan in which (4-25 \% SD) was observed between kit and AAS results [16].

\subsection{Contour Mapping}

In Fig. 3 Contour plot of As concentration (ppb) in ground water is shown by the map of Sindh province,

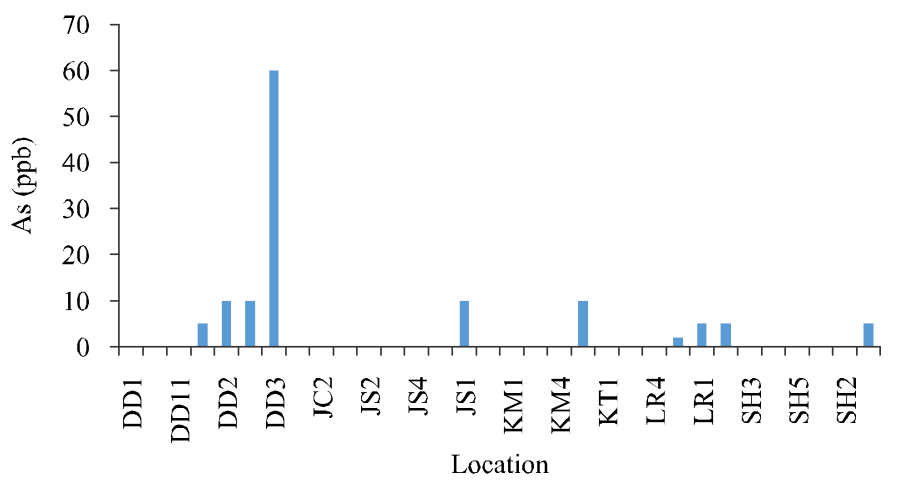

FIG. 1. SAMPLES ANALYZED FROM THE AREAS OF THE RIGHT BANK OF RIVER INDUS THROUGH ARSENIC KIT METHOD

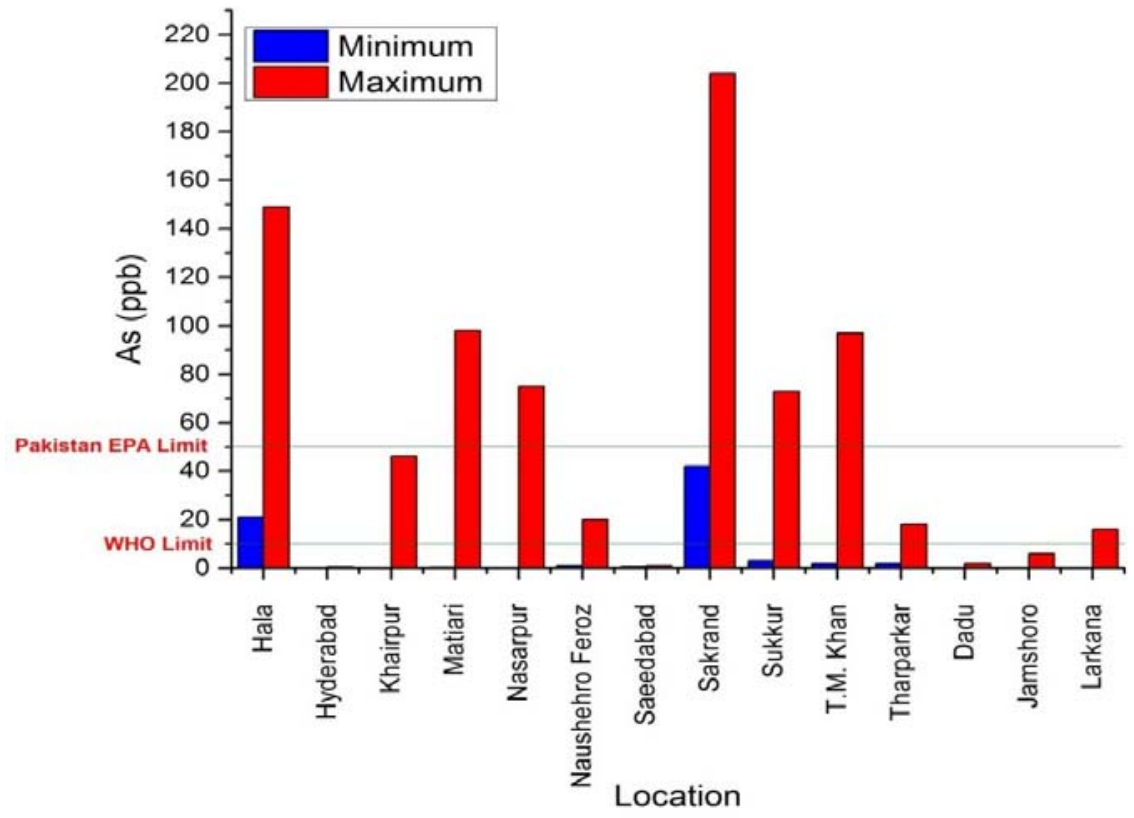

FIG. 2. ARSENIC IN SINDH SHOWING MINIMUM AND MAXIMUM CONCENTRATIONS WITH (WHO) AND (PAKISTAN- EPA LIMITS)

Mehran University Research Journal of Engineering \& Technology, Volume 36, No. 4, October, 2017 [p-ISSN: 0254-7821, e-ISSN: 2413-7219] 
Pakistan. The contour map was made by using sample locations (coordinates) on google maps and OriginPro 9 software. The black dots are showing the sampling locations at various regions of Sindh province. The cross-sectional lines show the non-sampling areas in the map. The As concentration is presented in the map by various colors according to the As ranges shown along with the contour map. It is evident from the Fig. 3 that As was found in higher concentrations on the left bank of RI. The red color highlights the highest As levels at the location MajeedKeerio, SakrandTaluka, district Shaheed Benazirabad. The other higher As contaminations are displayed by orange, yellow, green and cyan colors at Hala, Matairi, TMK and Nasarpur. The authors discussed the ground water samples As (ppb) in district Rahim Yar Khan, Punjab, Pakistan with the help of contour diagrams. They found As levels from 8-118 ppb in ground water of district Rahim Yar

TABLE 2. COMPARISON OF ARSENIC CONCENTRATION ANALYZED WITH KIT METHOD AND ATOMIC ABSORPTION SPECTROMETER

\begin{tabular}{|c|c|c|c|}
\hline Sample Code & As (ppb) Kit Method & As (ppb) with AAS & \% Standard Deviation $( \pm)$ \\
\hline HL4 & 50 & 37 & 9.192 \\
\hline HL10 & 40 & 23 & 12.020 \\
\hline HL11 & 60 & 39 & 14.849 \\
\hline HL12 & 50 & 34 & 11.313 \\
\hline HL14 & 50 & 38 & 8.485 \\
\hline HL20 & 50 & 70 & 14.142 \\
\hline HL21 & 55 & 40 & 10.606 \\
\hline HL22 & 40 & 40 & 0 \\
\hline HL24 & 70 & 43 & 19.091 \\
\hline HL27 & 50 & 36 & 9.899 \\
\hline HL28 & 100 & 96 & 2.828 \\
\hline HL41 & 70 & 51 & 13.435 \\
\hline KP6 & 25 & 25 & 0 \\
\hline KP16 & 46 & 45 & 0.707 \\
\hline MT11 & 30 & 16 & 9.899 \\
\hline MT12 & 80 & 53 & 19.091 \\
\hline MT14 & 110 & 98 & 8.485 \\
\hline MT17 & 5 & 3 & 1.414 \\
\hline NP2 & 40 & 44 & 2.828 \\
\hline NP3 & 35 & 35 & 0 \\
\hline NP7 & 60 & 57 & 2.121 \\
\hline NP10 & 80 & 75 & 3.535 \\
\hline NF9 & 15 & 18 & 2.121 \\
\hline NF10 & 13 & 20 & 4.949 \\
\hline PA5 & 30 & 20 & 7.071 \\
\hline PA12 & 90 & 73 & 12.020 \\
\hline SK5 & 35 & 42 & 4.949 \\
\hline SR3 & 25 & 20 & 3.535 \\
\hline TM6 & 5 & 9 & 2.828 \\
\hline TM7 & 100 & 97 & 2.121 \\
\hline TM9 & 15 & 2 & 9.192 \\
\hline TM10 & 50 & 70 & 14.142 \\
\hline LR1 & 5 & 1 & 2.828 \\
\hline
\end{tabular}

Mehran University Research Journal of Engineering \& Technology, Volume 36, No. 4, October, 2017 [p-ISSN: 0254-7821, e-ISSN: 2413-7219] 
Khan, which is located on the left bank of RI [16]. Another study conducted in the Shorabhaji region, northwest of Iran shows the arsenic contents of 1000 $\mathrm{ppb}$. The authors used geochemical contour mapping to determine As and other elements and found As in both metallic and non-metallic forms [17].

\subsection{Contamination Index $\left(\mathrm{C}_{\mathrm{d}}\right)$}

It is a method to evaluate and anticipate the regions characterized by irregular or toxic contents of elements. This shows the extent of contamination by taking into consideration the number of parameters crossing the upper allowable levels of hazardous elements and concentrations above these levels. The equation of calculating $\mathrm{C}_{\mathrm{d}}$ is as follows [18]:

$\mathrm{C}_{\mathrm{d}}=\sum_{\mathrm{i}=1}^{\mathrm{n}} \mathrm{C}_{\mathrm{fi}}$
Where,

$\mathrm{C}_{\mathrm{fi}}=\mathrm{C}_{\mathrm{Ai}} / \mathrm{C}_{\mathrm{Ni}}-1$

$\mathrm{C}_{\mathrm{fi}}$ is the contamination factor for $\mathrm{i}^{\text {th }}$ component, $\mathrm{C}_{\mathrm{Ai}}$ is the analytical value of $\mathrm{i}^{\text {th }}$ component, and $\mathrm{C}_{\mathrm{Ni}}$ is the upper allowable concentration of $\mathrm{i}^{\text {th }}$ component.

In this study, $\mathrm{C}_{\mathrm{d}}$ is studied for As. Contamination index for ground water was in the range between $0-19.61 .5 \%$ of the samples were below the permissible limit of WHO guidelines. The ground water on the basis of $C_{d}$ values may be arranged into four categories; $8.94 \%$ of ground water contained $\mathrm{C}_{\mathrm{d}}<1$ showed low contamination, $12.85 \%$ were in the range $1<\mathrm{C}_{\mathrm{d}}<3$ expressing intermediate contamination, $13.97 \%$ were within $3<\mathrm{C}_{\mathrm{d}}<10$ and $2.79 \%$ samples resulted in $\mathrm{C}_{\mathrm{d}}>10$ demonstrating the very high contamination. The ground water samples on the left bank

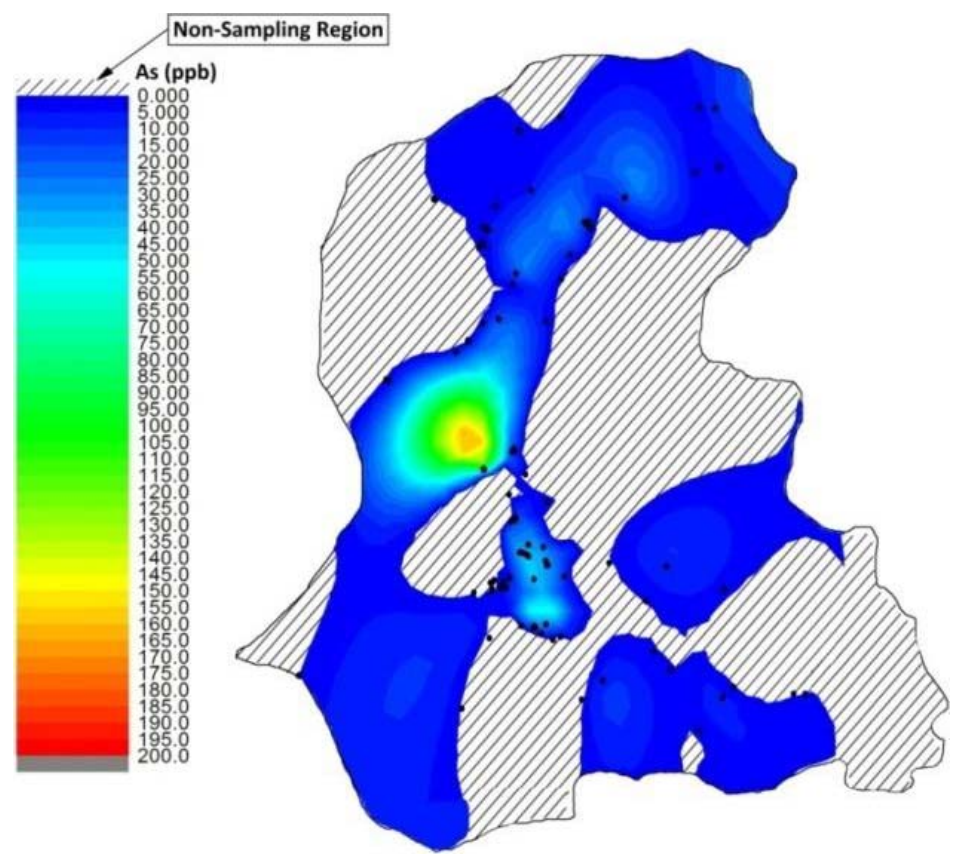

FIG. 3. CONTOUR PLOT OF ARSENIC CONCENTRATION (PPB) FROM GROUND WATER IN SINDH PROVINCE, PAKISTAN AT SELECTED SAMPLING LOCATIONS (BLACK DOTS SHOW SAMPLING LOCATIONS, CROSS- SECTIONAL LINES SHOW THE REGIONS FROM WHERE SAMPLES WERE NOT COLLECTED)

Mehran University Research Journal of Engineering \& Technology, Volume 36, No. 4, October, 2017 [p-ISSN: 0254-7821, e-ISSN: 2413-7219] 
Determination of Arsenic and Health Risk Assessment in the Ground Water of Sindh, Pakistan

TABLE 3. HEALTH RISK ASSESSMENT THROUGH GROUND WATER CONSUMPTION OF REGIONS LOCATED ON LEFT BANK OF RIVER INDUS IN SINDH PROVINCE

\begin{tabular}{|c|c|c|c|c|c|c|}
\hline No. & Location Code & Location & As Contamination (?g/L) & EDI (?g/kg-day) & THQ & CR \\
\hline 1. & HL3 & \multirow{16}{*}{ Hala } & 21 & 0.583 & $1.94 \mathrm{E}+00$ & $8.75 E+02$ \\
\hline 2. & HL10 & & 23 & 0.638 & $2.13 \mathrm{E}+00$ & $9.58 \mathrm{E}+02$ \\
\hline 3. & HL12 & & 34 & 0.944 & $3.15 \mathrm{E}+00$ & $1.42 \mathrm{E}+03$ \\
\hline 4. & HL27 & & 36 & 1 & $3.33 \mathrm{E}+00$ & $1.50 \mathrm{E}+03$ \\
\hline 5. & HL40 & & 36 & 1 & $3.33 \mathrm{E}+00$ & $1.50 \mathrm{E}+03$ \\
\hline 6. & HL4 & & 37 & 1.027 & $3.43 \mathrm{E}+00$ & $1.54 \mathrm{E}+03$ \\
\hline 7. & HL14 & & 38 & 1.055 & $3.52 \mathrm{E}+00$ & $1.58 \mathrm{E}+03$ \\
\hline 8. & HL11 & & 39 & 1.083 & $3.61 \mathrm{E}+00$ & $1.63 \mathrm{E}+03$ \\
\hline 9. & HL21 & & 40 & 1.111 & $3.70 \mathrm{E}+00$ & $1.67 \mathrm{E}+03$ \\
\hline 10. & HL22 & & 40 & 1.111 & $3.70 \mathrm{E}+00$ & $1.67 \mathrm{E}+03$ \\
\hline 11. & HL24 & & 43 & 1.194 & $3.98 \mathrm{E}+00$ & $1.79 \mathrm{E}+03$ \\
\hline 12. & HL41 & & 51 & 1.416 & $4.72 \mathrm{E}+00$ & $2.13 \mathrm{E}+03$ \\
\hline 13. & HL20 & & 70 & 1.944 & $6.48 \mathrm{E}+00$ & $2.92 \mathrm{E}+03$ \\
\hline 14. & HL28 & & 96 & 2.666 & $8.89 \mathrm{E}+00$ & $4.00 \mathrm{E}+03$ \\
\hline 15. & HL47 & & 101 & 2.805 & $9.35 \mathrm{E}+00$ & $4.21 \mathrm{E}+03$ \\
\hline 16. & HL16 & & 149 & 4.138 & $1.38 \mathrm{E}+01$ & $6.21 \mathrm{E}+03$ \\
\hline 17. & KP6 & \multirow{2}{*}{ Khairpur } & 25 & 0.694 & $2.31 \mathrm{E}+00$ & $1.04 \mathrm{E}+03$ \\
\hline 18. & KP16 & & 46 & 1.277 & $4.26 \mathrm{E}+00$ & $1.92 \mathrm{E}+03$ \\
\hline 19. & MT8 & \multirow{6}{*}{ Matiari } & 11 & 0.305 & $1.02 \mathrm{E}+00$ & $4.58 \mathrm{E}+02$ \\
\hline 20. & MT11 & & 16 & 0.444 & $1.48 \mathrm{E}+00$ & $6.67 \mathrm{E}+02$ \\
\hline 21. & MT12 & & 53 & 1.472 & $4.91 \mathrm{E}+00$ & $2.21 \mathrm{E}+03$ \\
\hline 22. & MT13 & & 67 & 1.861 & $6.20 \mathrm{E}+00$ & $2.79 E+03$ \\
\hline 23. & MT15 & & 75 & 2.083 & $6.94 \mathrm{E}+00$ & $3.13 E+03$ \\
\hline 24. & MT14 & & 98 & 2.722 & $9.07 \mathrm{E}+00$ & $4.08 \mathrm{E}+03$ \\
\hline 25. & NP3 & \multirow{6}{*}{ Nasarpur } & 35 & 0.972 & $3.24 \mathrm{E}+00$ & $1.46 \mathrm{E}+03$ \\
\hline 26. & NP2 & & 44 & 1.222 & $4.07 \mathrm{E}+00$ & $1.83 \mathrm{E}+03$ \\
\hline 27. & NP9 & & 56 & 1.555 & $5.19 \mathrm{E}+00$ & $2.33 \mathrm{E}+03$ \\
\hline 28. & NP7 & & 57 & 1.583 & $5.28 \mathrm{E}+00$ & $2.38 \mathrm{E}+03$ \\
\hline 29. & NP6 & & 66 & 1.833 & $6.11 \mathrm{E}+00$ & $2.75 \mathrm{E}+03$ \\
\hline 30. & NP10 & & 75 & 2.083 & $6.94 \mathrm{E}+00$ & $3.13 \mathrm{E}+03$ \\
\hline 31. & NF4 & \multirow{2}{*}{ Naushahro Feroz } & 18 & 0.5 & $1.67 \mathrm{E}+00$ & $7.50 \mathrm{E}+02$ \\
\hline 32. & NF3 & & 20 & 0.555 & $1.85 \mathrm{E}+00$ & $8.33 \mathrm{E}+02$ \\
\hline 33. & PA7 & \multirow{5}{*}{ Pano Aqil } & 11 & 0.305 & $1.02 \mathrm{E}+00$ & $4.58 \mathrm{E}+02$ \\
\hline 34. & PA5 & & 20 & 0.555 & $1.85 \mathrm{E}+00$ & $8.33 \mathrm{E}+02$ \\
\hline 35. & PA3 & & 27 & 0.75 & $2.50 \mathrm{E}+00$ & $1.13 \mathrm{E}+03$ \\
\hline 36. & PA4 & & 37 & 1.027 & $3.43 \mathrm{E}+00$ & $1.54 \mathrm{E}+03$ \\
\hline 37. & PA1 & & 73 & 2.027 & $6.76 \mathrm{E}+00$ & $3.04 \mathrm{E}+03$ \\
\hline 38. & SK5 & \multirow{3}{*}{ Sakrand } & 42 & 1.166 & $3.89 \mathrm{E}+00$ & $1.75 \mathrm{E}+03$ \\
\hline 39. & SK2 & & 195 & 5.416 & $1.81 \mathrm{E}+01$ & $8.13 \mathrm{E}+03$ \\
\hline 40. & SK8 & & 204 & 5.666 & $1.89 \mathrm{E}+01$ & $8.50 \mathrm{E}+03$ \\
\hline 41. & ST1 & \multirow{3}{*}{ Sekhat } & 18 & 0.5 & $1.67 \mathrm{E}+00$ & $7.50 \mathrm{E}+02$ \\
\hline 42. & ST1 & & 49 & 1.361 & $4.54 \mathrm{E}+00$ & $2.04 \mathrm{E}+03$ \\
\hline 43. & SK3 & & 20 & 0.555 & $1.85 \mathrm{E}+00$ & $8.33 E+02$ \\
\hline 44. & TM14 & \multirow{3}{*}{ Tando Muhammad Khan } & 27 & 0.75 & $2.50 \mathrm{E}+00$ & $1.13 \mathrm{E}+03$ \\
\hline 45. & TM10 & & 70 & 1.944 & $6.48 \mathrm{E}+00$ & $2.92 \mathrm{E}+03$ \\
\hline 46. & TM7 & & 97 & 2.694 & $8.98 \mathrm{E}+00$ & $4.04 \mathrm{E}+03$ \\
\hline 47. & TP3 & Tharparkar & 18 & 0.5 & $1.67 \mathrm{E}+00$ & $7.50 \mathrm{E}+02$ \\
\hline \multicolumn{3}{|c|}{ Average } & 53.702 & 1.492 & 4.972 & 2237.589 \\
\hline \multicolumn{3}{|c|}{ Minimum } & 11 & 0.305 & 1.018 & 458.333 \\
\hline \multicolumn{3}{|c|}{ Maximum } & 204 & 5.667 & 18.889 & 8500 \\
\hline \multicolumn{3}{|c|}{ Safe Values } & $10(\mu \mathrm{g} / \mathrm{L})$ (WHO Guideline) & - & 1 & 1 in $10^{-6}$ \\
\hline
\end{tabular}

Mehran University Research Journal of Engineering \& Technology, Volume 36, No. 4, October, 2017 [p-ISSN: 0254-7821, e-ISSN: 2413-7219] 
of RI have elevated $C_{d}$ values because of the higher contents of As in ground water. Mahar et. al. [16] also reported that riverine areas of district Rahim Yar Khan, which is located on the border of Sindh and Punjab provinces, Pakistan were contaminated with As and other impurities.

\subsection{Health Risk Assessment}

The HRA of the left bank RI was performed because it was found more contaminated than the right bank in this study. The people of the studied area consume ground water for their drinking, household and farming purposes. The residents of these areas cannot afford mineral water, therefore, they utilize ground water for their daily use. The estimated daily intake EDI ( $\mu \mathrm{g} / \mathrm{kg}$-day) for As is given in Table 3.

EDI values ranged from 0.305-5.667 ( $\mu \mathrm{g} / \mathrm{kg}$-day) for As. The highest EDI value of 5.66 ( $\mu \mathrm{g} / \mathrm{kg}$-day) was observed in the sample of Sakrand district showing the elevated threat level of As contamination to the local people. The higher EDI values of As can be because of municipal waste, leaching of waste materials in the groundwater, use of excess fertilizers and pesticides by farmers.

The THQ indices were calculated to get a health risk assessment. THQ index for As in potable water was in the range from 1.018-18.889. The results show that the THQ is crossing the safe limits, i.e. (THQ should be less than 1) posing a high health risk to the inhabitants of examined areas. Around, 90\% population of the left bank $\mathrm{RI}$ is utilizing ground water for their domestic and drinking purposes, hence at high health risk because of As contamination which is "carcinogenic to human beings".
As is the single available element in the periodic table for which there is a cancer slope factor.CR was calculated in the ground water as shown in Table 3 . The CR values ranged from 458.333-8500 with an average value of 2237.589 in the ground water of examined region exhibiting elevated risk. It is reported that CR value 1 in $10^{6}$ is significantly considered [12,19]. The results of this study show that the ground water exhibits high risk in the studied areas of Sindh province when compared to USEPA (2010) guidelines. The cancer risk assessment results of this work show the elevated health risk in the observed areas of Sindh.

\section{CONCLUSION}

The kit method was used to analyze the 214 ground water samples from the both sides of RI. Then AAS technique was applied to analyze 83 samples from the left bank of RI. The high THQ and CR values for As indicated, that higher intake of As in ground water may pose chronic toxicity and carcinogenic risk to the local inhabitants.

Our results suggest that the ground water from the left bank of RI areas i.e. Sakrand, Hala, Matiari, TMK, Nasarpur and Sukkur is not safe for drinking without proper treatment.

Hence drinking water quality and continuous assessment should be conducted at regular intervals to check the quality of drinking water.

As kit and atomic absorption spectrometer results are quite similar with $0.707-19 \% \mathrm{SD}$. The contour plot of As concentration (ppb) in ground water is shown by the map of Sindh and it is observed that the higher levels of arsenic are present on the left bank of RI. Contamination index was calculated for ground water of Sindh and found in the range between 0-19. 


\section{ACKNOWLEDGMENT}

This project is funded by Pakistan-US Science \& Technology Cooperation Program, and Higher Education Commission, Pakistan. The authors are thankful to students of the Department of Chemical Engineering, Mehran University of Engineering \& Technology, Jamshoro, for their help in the ground water sample collection.

\section{REFERENCES}

Kazi, T.G., Arain, M.B., Baig, J.A., Jamali, M.K., Afridi, in Drinking Water with the Biological Samples of Skin Disorders”, Science of the Total Environment, Volume 407, No.3, pp. 1019-1026, 2009.

Martínez-Barquero, V., de Marco, G., Martínez-Hervas, S., Rentero, P., Galan-Chilet, I., and Blesa, S., "Polymorphisms in Endothelin System Genes, Arsenic Levels and Obesity Risk”, Volume 10, No. 3, pp 1-13, 2015.

Baig, J.A., Kazi, T.G., Shah, A.Q., Afridi, H.I., Khan, S., and Kolachi, N.F., "Evaluation of Toxic Risk Assessment of Arsenic in Male Subjects through Drinking Water in Southern Sindh, Pakistan”, Biological Trace Element Research, Volume 143, No. 2, pp. 772-786, 2011.

Abernathy, C.O., Thomas, D.J., and Calderon, R.L., "Health Effects and Risk Assessment of Arsenic", The Journal of Nutrition, Volume 133, No. 5, pp. 1536-1538, 2003.

Arain, M., Kazi, T.G., Baig, J.A., Jamali, M., Afridi, H.I., and Shah, A., "Determination of Arsenic Levels in Lake Water, Sediment, and Foodstuff from Selected Area of Sindh, Pakistan: Estimation of Daily Dietary Intake”, Food and Chemical Toxicology, Volume 47, No. 1, pp. 242-248, 2009.

[6]

Husain, V., Niazam, H., and Arain, G.M., "Arsenic and H.I., and Jalbani, N., "The Correlation of Arsenic Levels

Zhang, W., Singh, P., Paling, E., and Delides S., “Arsenic Removal from Contaminated Water by Natural Iron Ores”, Minerals Engineering, Volume 17, No. 4, pp. 517-524, 2004. Fluoride Mobilization Mechanism in Groundwater of Indus Delta and Thar Desert, Sindh, Pakistan”, International Journal of Economic and Environmental Geology, Volume 3, pp. 15-23, 2012.
Zhang, Q.L., Lin, Y., Chen, X., and Gao N.Y., “A Method for Preparing Ferric Activated Carbon Composites Sdsorbents to Remove Arsenic from Drinking Water”, Journal of Hazardous Materials, Volume 148, No. 3, pp. 671-678, 2007.

Sahin, C.A., Tokgöz, Ý., and Bektap, S., "Preconcentration and Determination of Iron and Copper in Spice Samples by Cloud Point Extraction and Flow Injection Flame Atomic Absorption Spectrometry”, Journal of Hazardous Materials, Volume 181, No. 1, pp. 359-365, 2010.

Moya, J., Phillips, L., Schuda, L., Wood, P., Diaz, and Lee, A.R., "Exposure Factors Handbook", US Environmental Protection Agency, Washington, 2011.

Rasool, A., Farooqi, A., Xiao, T., Masood, S., and Kamran, M.A., "Elevated Levels of Arsenic and Trace Metals in Drinking Water of Tehsil Mailsi, Punjab, Pakistan”, Journal of Geochemical Exploration, Volume 169, pp. 89-99, 2016.

Rasool, A., Xiao, T., Farooqi, A., Shafeeque, M., Masood, S., and Ali, S., "Arsenic and Heavy Metal Contaminations in the Tube Well Water of Punjab, Pakistan and Risk Assessment: A Case Study”, Ecological Engineering, Volume 95, pp. 90-100, 2016.

USEPARA Forum, "Guidelines for Carcinogen Risk Assessment”, 2005.

Baig, J.A., Kazi, T.G., Mustafa, M.A., Solangi, I.B., Mughal, M.J., and Afridi, H.I., "Arsenic Exposure in Children through Drinking Water in Different Districts of Sindh, Pakistan”, Biological Trace Element Research, pp. 1-12, 2016.

[15] Baig, J.A., Kazi, T.G., Arain, M.B., Afridi, H.I., Kandhro, G.A., and Sarfraz, R.A., "Evaluation of Arsenic and other Physico-Chemical Parameters of Surface and Ground Water of Jamshoro, Pakistan”, Journal of Hazardous Materials, Volume 166, No. 2, pp. 662-669, 2009. 
[16] Mahar, M.T., Khuhawar, M.Y., Jahangir, T.M., and Baloch, M.A., "Determination of Arsenic Contents in Groundwater of District Rahim Yar Khan Southern Punjab, Pakistan”, Arabian Journal of Geosciences, Volume 8, No. 12, pp. 10983-10994, 2015.

[17] Daya, A.A., “Application of Concentration-Area Method for Separating Geochemical Anomalies from Background: ACase Study of Shorabhaji Region, Northwest of Iran”, Arabian Journal of Geosciences, Volume 8, No. 6, pp. 3905-3913, 2015.
[18] Backman, B., Bodiš, D., Lahermo, P., Rapant, S., and Tarvainen, T., "Application of a Groundwater Contamination Index in Finland and Slovakia”, Environmental Geology, Volume 36, No. 1-2, pp. 5564, 1998.

[19] Fewtrell, L., and Bartram, J., "Water Quality: Guidelines, Standards and Health: Assessment of Risk and Risk Management for Water-Related Infectious Diseases”, 2001. 